\title{
Density functional theory in the canonical ensemble: I General formalism
}

\author{
J A Hernando \\ Department of Physics, Comisión Nacional de Energía Atómica, \\ Av. del Libertador 8250, 1429 Buenos Aires, Argentina
}

\begin{abstract}
Density functional theory stems from the Hohenberg-Kohn-Sham-Mermin (HKSM) theorem in the grand canonical ensemble (GCE). However, as recent work shows, although its extension to the canonical ensemble (CE) is not straightforward, work in nanopore systems could certainly benefit from a mesoscopic DFT in the CE. The stumbling block is the fixed $N$ constraint which is responsible for the failure in proving the interchangeability of density profiles and external potentials as independent variables. Here we prove that, if in the $\mathrm{CE}$ the correlation functions are stripped off of their asymptotic behaviour (which is not in the form of a properly irreducible $n$ body function), the HKSM theorem can be extended to the CE. In proving that, we generate a new hierarchy of $N$-modified distribution and correlation functions which have the same formal structure that the more conventional ones have (but with the proper irreducible $n$-body behaviour) and show that, if they are employed, either a modified external field or the density profiles can indistinctly be used as independent variables. We also write down the $N$-modified free energy functional and prove that the thermodynamic potential is minimized by the equilibrium values of the new hierarchy.

PACS numbers: 61.20.Gy, 68.45.-v, 05.20.-y
\end{abstract}




\section{Introduction}

Density functional theory (DFT) has become, since the seminal work of Hohenberg, Kohn and Sham [1] and its extension to the grand canonical ensemble (GCE) by Mermin [2], one of the more widely used techniques in condensed matter theory. It has been used in a great variety of systems [3, 4] as, e.g., uniform and non uniform systems in simple [5, 6] and general [7] fluids, confined fluids [8, 9], melting and freezing [10], interfaces [11], etc. as well as in the calculation of electronic properties of all sort of systems [12]. This impressive amount of work has been spawned by the Hohenberg-Kohn-ShamMermin (HKSM) theorem which, roughly speaking, states that, either the external potential or the density profile can indistinctly be used as independent variables and that the thermodynamic grand potential reaches its minimum value when the equilibrium density profile is used. More precisely, it has been proved [13 that any positive density can be viewed as an equilibrium density for a system in a suitable external potential. Therefore, it is in the foundation of all sort of variational principles in the GCE. On the other hand, the failure to implement a straightforward canonical ensemble (CE) extension is already well known, referred to in [9] (where a GCE series expansion was done and an approximate density profile for the $\mathrm{CE}$ obtained), can be traced back to the fixed $\mathrm{N}$ constraint (see Section 2) and it manifests itself in the failure of having an Ornstein-Zernike (OZ) equation. In fact, years ago, Ramshaw [14 explicitly worked out the two-body OZ equation for a one component system, showing how the stripping of the correlation function $h(r)$ off its asymptotic behaviour solves the problems found in a more conventional approach. Another approach to a DFT in the CE, equivalent to the one given in [9], is discussed in [15]. In [16] the existence of an extension of the $\mathrm{OZ}$ equation for the $\mathrm{CE}$ is discussed in the framework of a DFT theory. As the GCE is an extension of the CE, one may wonder about the need of an explicit CE formulation. The problem addressed by that question is about the equivalence between both ensembles that holds true in the thermodynamic limit but breaks down for small systems. There is a great body of work done on the properties of fluids confined in very small cavities which can shrink down to sizes that hold only a few adsorbed molecules. Experiments done in porous glasses (with a mean pore radius $r \approx 20-30 \AA$ ) [17] have shown how such a degree of confinement induces strong changes with respect to bulk properties [10, 18] as, e.g., critical point shifting [7], capillary condensation [19], freezing point decreasing [20], etc. Earlier approaches to these highly inhomogeneous systems included virial expansions adapted to this situation [21, 22], calculations of the partition function [22, 23] and mean field theory [24]. Numerical simulation techniques have been also used to study adsorption [25], phase transitions [20], [26, 27], etc. Within the framework of DFT, the most successful DFT that deals with inhomogeneous systems is Rosenfeld's fundamental measure functional (FMF) [6] which, in particular, has been used in some of the GCE expansions referred to above ([9], 15]) in order to obtain a CE expansion. It is quite clear, then, the interest of having a mesoscopic DFT in the CE. Very recently we have also presented, for one component systems, [28] an extension of 
the HKSM theorem to the CE.

In this paper we prove the results put forward in [28] and extend them to general mixtures. The system we consider is a $p$ components mixture (labeled by greek indices and interacting between themselves with two-body potentials), each $\alpha$ component with $N_{\alpha}$ particles in a box of volume $V$ and under the influence of one body, species dependent, external potentials $V_{\alpha}^{(1)}(\mathbf{x})$. We first extend Ramshaw's work 14 not only by considering mixtures but, fundamentally, by proving that by stripping the canonical correlation functions off of their asymptotic behaviour an $N$-modified set of distribution and correlation function hierarchies is generated. Specifically, we prove that they can be defined by functional derivatives of the canonical partition function $Q_{N}$ with respect to a modified external potential and, moreover, that the $n$-body direct and total correlation functions are related through $n$-body OZ equations. The physical idea is the same that shows up in almost every textbook on Statistical Mechanics when going from the CE to the GCE. There an open subset is considered, here we strip the canonical correlation functions off their asymptotic behaviour. The difference is that, for us, this is the fundamental step, not an intermediate one. We are then able to prove that this step is described, to any $n$-body order, by functional derivatives with the right formal structure. We then prove the HKSM theorem in the CE by introducing an $N$-modified free energy and thermodynamic potential which is minimized by the $N$-modified density profiles. In this way, variational principles formulated in the CE have a firm foundation if the $N$-modified functions are used in them. Let us emphasize that, as any successful theory of liquids needs to consider all the correlation functions (which must have the correct hierarchical structure), a valid OZ equation for the pair functions $h$ and $c$ is not enough to extend DFT to the CE. Therefore, one of the crucial points of this paper is the proof of the existence of the $N$-modified hierarchies, that they are related by the usual formal scheme and are linked by $n$-body OZ equations. As side remarks, let us mention that i) the two-body interaction potential restriction can be also lifted by including more than one-body external potentials [29 but, for the sake of clarity, we will restrict ourselves to the two-body interaction potentials case and ii) there exists at least one system (lattice gases) where the conventional CE approach fails (due to the fixed topology constraint), even in the thermodynamic limit, and it will be discussed in a forthcoming paper.

The layout of the paper is as follows: in Section 2 we discuss the reasons behind the failure of a conventional CE approach, finite size effects in the pair function $h(r)$ are discussed in Section 3, while in Section 4 it is proved that, if the (already discussed) asymptotic behaviour of the pair canonical correlation functions is removed, then a twobody OZ equation in the CE can be formulated. Section 5 discusses both the generation of the $N$-modified distribution and correlation function hierarchies and of the set of $n$ body OZ equations that relate them while, in Section 6 , we introduce an $N$-modified free energy, write down the variational equations for the density profiles and show that the associated $N$-modified grand thermodynamic potential is minimized by the equilibrium density profiles. This completes the extension of the HKSM theorem to the CE and, in this way, variational principles formulated in the CE have a firm foundation if the 
$N$-modified functions are used in them. Section 7 summarizes our results.

\section{Failure of the canonical approach}

The system we consider is a $p$ components general mixture where the species are labeled by greek indices $\alpha=1, \ldots, p$, each one of them has $N_{\alpha}$ particles (indicated by $k_{\alpha}=1, \ldots, N_{\alpha}$ ) which are under the influence of one-body species-dependent external fields $V_{\alpha}^{(1)}(\mathbf{r})$ that can be varied at will and the box volume is $V$. The canonical partition function is

$$
Q(\{\mathbf{N}\}, V, T)=\frac{1}{\mathbf{N} ! V^{\mathbf{N}}} \int \mathrm{e}^{H_{0}+\sum_{\nu, k_{\nu}} \Phi_{\nu}\left(\mathbf{r}_{k \nu}\right)} \mathrm{d}\{\mathbf{r}\}
$$

Here $\{\mathbf{r}\}$ denotes the configuration space of all the particles, i.e. $\mathrm{d}\{\mathbf{r}\}=\prod_{\alpha k_{\alpha}} \mathrm{d}\left\{\mathbf{r}_{k_{\alpha}}\right\}$, $\mathbf{N} !=\prod_{\alpha=1}^{p} N_{\alpha}$ !, and a $-\beta(=-1 / k T)$ factor has been absorbed in the hamiltonian and fields. In particular, $H_{0}$ is the system's hamiltonian in the absence of external fields (contains all the two-body interaction terms) and $\Phi_{\alpha}(\mathbf{r})=-\beta V_{\alpha}^{(1)}(\mathbf{r})$. The one particle canonical distribution function is usually defined by [30]

$$
\begin{aligned}
n_{\alpha}^{(1)}(\mathbf{x}) & =\left\langle\sum_{k_{\alpha}} \delta\left(\mathbf{x}-\mathbf{r}_{k_{\alpha}}\right)\right\rangle=\frac{\int \sum_{k_{\alpha}} \delta\left(\mathbf{x}-\mathbf{r}_{k_{\alpha}}\right) \mathrm{e}^{H_{0}+\sum_{\nu, k_{\nu}} \Phi_{\nu}\left(\mathbf{r}_{k \nu}\right)} \mathrm{d}\{\mathbf{r}\}}{Q_{\mathbf{N}}}= \\
& =\frac{\mathrm{e}^{\Phi_{\alpha}(\mathbf{x})}}{Q_{\mathbf{N}}} \frac{\delta Q_{\mathbf{N}}}{\delta \mathrm{e}^{\Phi_{\alpha}(\mathbf{x})}}
\end{aligned}
$$

and can also be written as

$$
n_{\alpha}^{(1)}(\mathbf{x})=\frac{1}{Q_{\mathbf{N}}} \frac{\delta Q_{\mathbf{N}}}{\delta \Phi_{\alpha}(\mathbf{x})}=\hat{n}_{\alpha}^{(1)}(\mathbf{x})
$$

Both $n_{\alpha}^{(1)}(\mathbf{x})$, the conventional canonical distribution function, and $\hat{n}_{\alpha}^{(1)}(\mathbf{x})$, the full canonical distribution function (as defined by Lebowitz and Percus [31]), are the first members of two hierarchies of distribution functions. The s-body functions of these hierarchies can be generated by functional derivation

$$
\begin{aligned}
& n_{\boldsymbol{\alpha}}^{(s)}(\{\mathbf{x}\})=\frac{\prod_{k=1}^{s} \mathrm{e}^{\Phi_{\alpha_{k}}\left(\mathbf{x}_{k}\right)}}{Q_{\mathbf{N}}} \frac{\delta^{s} Q_{\mathbf{N}}}{\prod_{k=1}^{s} \delta \mathrm{e}^{\Phi_{\alpha_{k}}\left(\mathbf{x}_{k}\right)}} \\
& \hat{n}_{\boldsymbol{\alpha}}^{(s)}(\{\mathbf{x}\})=\frac{1}{Q_{\mathbf{N}}} \frac{\delta^{s} Q_{\mathbf{N}}}{\prod_{k=1}^{s} \delta \Phi_{\alpha_{k}}\left(\mathbf{x}_{k}\right)}
\end{aligned}
$$

Here $\boldsymbol{\alpha}=\left(\alpha_{1}, \ldots, \alpha_{s}\right),\{\mathbf{x}\}=\left(\mathbf{x}_{1}, \ldots, \mathbf{x}_{s}\right)$ are the species and coordinate sets and the difference between both hierarchies is that in the full one different coordinates can coincide, while in the other one can not. The GCE definition is formally the same with the grand partition function $\Xi$ replacing $Q_{N}$. The truncated correlation functions $t$ and $\hat{t}$ associated to these hierarchies and the direct correlation function $c$ are defined by 


$$
\begin{aligned}
& t_{\boldsymbol{\alpha}}^{(s)}(\{\mathbf{x}\})=\prod_{k=1}^{s} \mathrm{e}^{\Phi_{\alpha_{k}}\left(\mathbf{x}_{k}\right)} \frac{\delta^{s} \ln Q_{\mathbf{N}}}{\prod_{k=1}^{s} \delta \mathrm{e}^{\Phi_{\alpha_{k}}\left(\mathbf{x}_{k}\right)}} \\
& \hat{t}_{\boldsymbol{\alpha}}^{(s)}(\{\mathbf{x}\})=\frac{\delta^{s} \ln Q_{\mathbf{N}}}{\prod_{k=1}^{s} \delta \Phi_{\alpha_{k}}\left(\mathbf{x}_{k}\right)}=\frac{\delta \hat{t}_{\alpha_{1} \ldots \alpha_{s-1}}^{(s-1)}\left(\mathbf{x}_{1}, \ldots, \mathbf{x}_{s-1}\right)}{\delta \Phi_{\alpha_{s}}\left(\mathbf{x}_{s}\right)} \\
& c_{\boldsymbol{\alpha}}^{(s)}(\{\mathbf{x}\})=\beta \frac{\delta^{s} F^{(e x c)}}{\prod_{k=1}^{s} \delta n_{\alpha_{k}}^{(1)}\left(\mathbf{x}_{k}\right)}=\frac{\delta c_{\alpha_{1} \ldots \alpha_{s-1}}^{(s-1)}\left(\mathbf{x}_{1}, \ldots, \mathbf{x}_{s-1}\right)}{\delta n_{\alpha_{s}}^{(1)}\left(\mathbf{x}_{s}\right)}
\end{aligned}
$$

$F^{(e x c)}$ is the excess free energy. In particular, $t_{\alpha}^{(1)}(\mathbf{x})=\hat{t}_{\alpha}^{(1)}(\mathbf{x})=n_{\alpha}^{(1)}(\mathbf{x})=\hat{n}_{\alpha}^{(1)}(\mathbf{x})$ and as

$t_{\alpha_{1} \alpha_{2}}^{(2)}\left(\mathbf{x}_{1}, \mathbf{x}_{2}\right)=n_{\alpha_{1} \alpha_{2}}^{(2)}\left(\mathbf{x}_{1}, \mathbf{x}_{2}\right)-n_{\alpha_{1}}^{(1)}\left(\mathbf{x}_{1}\right) n_{\alpha_{2}}^{(1)}\left(\mathbf{x}_{2}\right)=n_{\alpha_{1}}^{(1)}\left(\mathbf{x}_{1}\right) n_{\alpha_{2}}^{(1)}\left(\mathbf{x}_{2}\right) h_{\alpha_{1} \alpha_{2}}^{(2)}\left(\mathbf{x}_{1}, \mathbf{x}_{2}\right)$

$\hat{t}_{\alpha_{1} \alpha_{2}}^{(2)}\left(\mathbf{x}_{1}, \mathbf{x}_{2}\right)=t_{\alpha_{1} \alpha_{2}}^{(2)}\left(\mathbf{x}_{1}, \mathbf{x}_{2}\right)+n_{\alpha_{1}}^{(1)}\left(\mathbf{x}_{1}\right) \delta_{\alpha_{1} \alpha_{2}} \delta\left(\mathbf{x}_{1}-\mathbf{x}_{2}\right)$

the link with the more usual notation is established. Also, up to now, the independent variables are the external fields. As by definition is $\hat{t}_{\alpha \lambda}^{(2)}(\mathbf{x}, \mathbf{y})=\delta n_{\alpha}^{(1)}(\mathbf{x}) / \delta \Phi_{\lambda}(\mathbf{y})$ the change in the density profiles due to changes in the external potentials is

$$
\delta n_{\alpha}^{(1)}(\mathbf{x})=\sum_{\lambda} \int \hat{t}_{\alpha \lambda}^{(2)}(\mathbf{x}, \mathbf{y}) \delta \Phi_{\lambda}(\mathbf{y}) \mathrm{d} \mathbf{y}
$$

If the density profiles $\left\{n_{\alpha}^{(1)}\right\}$ and the external potentials $\left\{\Phi_{\lambda}\right\}$ can interchangeably be used as independent variables, then an inverse kernel $\hat{t}_{\eta \alpha}^{(2)-1}(\mathbf{z}, \mathbf{x})\left(=\delta \Phi_{\eta}(\mathbf{z}) / \delta n_{\alpha}^{(1)}(\mathbf{x})\right)$ must necessarily exist such that

$$
\sum_{\alpha} \int \hat{t}_{\eta \alpha}^{(2)-1}(\mathbf{z}, \mathbf{x}) \hat{t}_{\alpha \lambda}^{(2)}(\mathbf{x}, \mathbf{y}) \mathrm{d} \mathbf{x}=\delta_{\eta \lambda} \delta(\mathbf{z}-\mathbf{y})
$$

In that case

$$
\delta \Phi_{\eta}(\mathbf{z})=\sum_{\alpha} \int \hat{t}_{\eta \alpha}^{(2)-1}(\mathbf{z}, \mathbf{x}) \delta n_{\alpha}^{(1)}(\mathbf{x}) \mathrm{d} \mathbf{x}
$$

When $\hat{t}_{\eta \alpha}^{(2)-1}$ is used to define the direct correlation function $c_{\eta \alpha}^{(2)}$ (see equation (37)) then the $\mathrm{OZ}$ equation follows immediately. Now we can prove that, in the CE, the inverse kernel $\hat{t}_{\eta \alpha}^{(2)-1}$ does not exist, while it does in the GCE. The normalization integrals are

$$
\begin{aligned}
& \int n_{\alpha}^{(1)}(\mathbf{x}) \mathrm{d} \mathbf{x}= \begin{cases}N_{\alpha} & \mathrm{CE} \\
\left\langle N_{\alpha}\right\rangle & \mathrm{GCE}\end{cases} \\
& \int n_{\alpha \lambda}^{(2)}(\mathbf{x}, \mathbf{y}) \mathrm{d} \mathbf{x} \mathrm{d} \mathbf{y}= \begin{cases}N_{\alpha}\left(N_{\lambda}-\delta_{\alpha \lambda}\right) & \mathrm{CE} \\
\left\langle N_{\alpha}\left(N_{\lambda}-\delta_{\alpha \lambda}\right)\right\rangle & \mathrm{GCE}\end{cases}
\end{aligned}
$$

and it is easy to show that

$$
\int n_{\lambda}^{(1)}(\mathbf{y})\left[1+h_{\alpha \lambda}^{(2)}(\mathbf{x}, \mathbf{y})\right] \mathrm{d} \mathbf{y}= \begin{cases}N_{\lambda}-\delta_{\alpha \lambda} & \mathrm{CE} \\ \frac{\partial \ln \left[\Xi n_{\alpha}^{(1)}(\mathbf{x}) / z_{\alpha}\right]}{\partial \ln z_{\lambda}} & \mathrm{GCE}\end{cases}
$$


It then follows that

$$
\int \hat{t}_{\alpha \lambda}^{(2)}(\mathbf{x}, \mathbf{y}) \mathrm{d} \mathbf{y}= \begin{cases}0 & \mathrm{CE} \\ n_{\alpha}^{(1)}(\mathbf{x})\left[\delta_{\alpha \lambda}+\frac{\partial \ln \left(n_{\alpha}^{(1)}(\mathbf{x}) / z_{\alpha}\right)}{\partial \ln z_{\lambda}}\right] & \mathrm{GCE}\end{cases}
$$

Equations (13) and (18) are obviously incompatible in the CE while, in the GCE and because of the fluctuations, they are not and the kernel $\hat{t}_{\alpha \lambda}^{(2)}$ is invertible. In consequence, the OZ equation (equations (37-38) in terms of the conventional correlation functions does not exist in the $\mathrm{CE}$. It is also clear that the existence of an OZ equation is a previous condition to the formulation of a DFT but, in fact, the equivalence must be established between the existence of the whole set of $n$-body OZ equations and DFT (see Sections 4 and 5).

\section{Finite size effects}

If a $\lambda$ particle is fixed in position $\mathbf{y}$, the conditional probability of finding an $\alpha$ particle in $\mathbf{x}$ is

$$
n_{\alpha \lambda}(\mathbf{x} \mid \mathbf{y})=\frac{n_{\alpha \lambda}^{(2)}(\mathbf{x}, \mathbf{y})}{n_{\lambda}^{(1)}(\mathbf{y})}=n_{\alpha}^{(1)}(\mathbf{x})\left[1+h_{\alpha \lambda}^{(2)}(\mathbf{x}, \mathbf{y})\right]
$$

This causes a density change that depends on $\{\lambda, \mathbf{y}\}$ which, when $\mathbf{x}$ and $\mathbf{y}$ are far away from each other, can also be written as

$$
n_{\alpha \lambda ; \infty}(\mathbf{x} \mid \mathbf{y})=n_{\alpha}^{(1)}(\mathbf{x})+\frac{\partial n_{\alpha}^{(1)}(\mathbf{x})}{\partial \rho_{\lambda}} \Delta \rho_{\lambda}(\mathbf{y})
$$

Here $\Delta \rho_{\lambda}(\mathbf{y})$ has the meaning of an unknown proportionality factor which, in fact, depends on all the $\{\alpha, \mathbf{x}\} ;\{\lambda, \mathbf{y}\}$ variables; it is not the difference in density that arises from fixing a $\lambda$ particle in $\mathbf{y}$. Therefore, the asymptotic behaviour of the total correlation function can be cast in the form (uncorrelated $\mathrm{x}-\mathrm{y}$ dependence)

$$
h_{\alpha \lambda ; \infty}^{(2)}(\mathbf{x}, \mathbf{y})=\frac{\partial \ln n_{\alpha}^{(1)}(\mathbf{x})}{\partial \rho_{\lambda}} \Delta \rho_{\lambda}(\mathbf{y})
$$

However, to follow this course of action (deriving with respect to the partial density $\rho_{\lambda}$ ) has, at least, two inconvenients: 1$)$ the $\{\alpha, \mathbf{x}\} \longleftrightarrow\{\lambda, \mathbf{y}\}$ exchange symmetry would be hidden and 2) the formalism would distinguish between diagonal $\left(h_{\alpha \alpha}^{(2)}\right)$ and non diagonal $\left(h_{\alpha \lambda}^{(2)}, \alpha \neq \lambda\right)$ correlation functions in the sense that the long range behavior of the latter cannot be easily separated (in equation (B3) the species and field indices would be different) and, therefore, the generalization that leads to $N$-modified hierarchy of distribution functions is far from clear. It can also be mentioned that, while stripping off the diagonal components is good enough for obtaining an invertible kernel, we preferred, for the reasons mentioned above, not to do that. On the other hand, it is known [32] that, in the $\mathrm{CE}$, the long range behaviour of the correlation functions is not in the nature 
of an irreducible two body function, it can be written in a separated variables form. Therefore, instead of equations (20-21) we write (redefining $\Delta \rho_{\lambda}(\mathbf{y})$ )

$$
h_{\alpha \lambda ; \infty}^{(2)}(\mathbf{x}, \mathbf{y})=\frac{\partial \ln n_{\alpha}^{(1)}(\mathbf{x})}{\partial \rho_{\alpha}} \Delta \rho_{\lambda}(\mathbf{y})
$$

and similarly, for $n_{\alpha \lambda ; \infty}$. Defining

$$
\begin{aligned}
& A_{\alpha}(\mathbf{x})=\frac{1}{\int \frac{\partial \ln n_{\alpha}^{(1)}(\mathbf{u})}{\partial \rho_{\alpha}} \mathrm{d} \mathbf{u}} \frac{\partial \ln n_{\alpha}^{(1)}(\mathbf{x})}{\partial \rho_{\alpha}} \\
& B_{\lambda}(\mathbf{y})=\frac{1}{\int \Delta \rho_{\lambda}(\mathbf{v}) \mathrm{d} \mathbf{v}} \Delta \rho_{\lambda}(\mathbf{y})
\end{aligned}
$$

Both $A_{\alpha}(\mathbf{x})$ and $B_{\lambda}(\mathbf{y})$ are normalized to one and $h_{\alpha \lambda ; \infty}^{(2)}(\mathbf{x}, \mathbf{y})$ can be written in two possible forms

$$
h_{\alpha \lambda ; \infty}^{(2)}(\mathbf{x}, \mathbf{y})=\tilde{\Gamma}_{\alpha \lambda} A_{\alpha}(\mathbf{x}) B_{\lambda}(\mathbf{y})=\Gamma_{\alpha \lambda} B_{\alpha}(\mathbf{x}) A_{\lambda}(\mathbf{y})
$$

Integrating over x we have $\tilde{\Gamma}_{\alpha \lambda} B_{\lambda}(\mathbf{y})=\Gamma_{\alpha \lambda} A_{\lambda}(\mathbf{y})$ and so

$$
\begin{aligned}
& h_{\alpha \lambda ; \infty}^{(2)}(\mathbf{x}, \mathbf{y})=\Gamma_{\alpha \lambda} \frac{\partial \ln n_{\alpha}^{(1)}(\mathbf{x})}{\partial \rho_{\alpha}} \frac{\partial \ln n_{\lambda}^{(1)}(\mathbf{y})}{\partial \rho_{\lambda}} \\
& \Gamma_{\alpha \lambda}=\int \frac{\partial \ln n_{\lambda}^{(1)}(\mathbf{u})}{\partial \rho_{\lambda}} \mathrm{d} \mathbf{u} \int \Delta \rho_{\alpha}(\mathbf{v}) \mathrm{d} \mathbf{v}
\end{aligned}
$$

$\Gamma_{\alpha \lambda}$ depends on $\{\alpha, \lambda\}$ and on the unknown function $\Delta \rho_{\alpha}(\mathbf{x})$. An $N$-modified canonical total correlation function $\tilde{h}_{\alpha \lambda}^{(2)}(N$-modified as implying that it is different from the correlation function $h_{\alpha \lambda}^{(2)}$ and that its definition is size-dependent) can then be defined by

$$
h_{\alpha \lambda}^{(2)}(\mathbf{x}, \mathbf{y})= \begin{cases}\tilde{h}_{\alpha \lambda}^{(2)}(\mathbf{x}, \mathbf{y})+\Gamma_{\alpha \lambda} \frac{\partial \ln n_{\alpha}^{(1)}(\mathbf{x})}{\partial \rho_{\alpha}} \frac{\partial \ln n_{\lambda}^{(1)}(\mathbf{y})}{\partial \rho_{\lambda}} & \mathbf{x} \neq \mathbf{y} \\ \tilde{h}_{\alpha \lambda}^{(2)}(\mathbf{x}, \mathbf{y})=-1 & \mathbf{x}=\mathbf{y}\end{cases}
$$

The $N$-modified correlation function $\tilde{h}_{\alpha \lambda}^{(2)}$ is the total correlation function stripped off of its asymptotic behaviour. Notice that, although this modification seems to affect all the $h^{(2)}$ values, the excluded volume effects (authentically two-body) are not modified, not only for the case $\mathbf{x}=\mathbf{y}$ but also because, as the stripping is done through a separation of variables, this ensures that only the long range behaviour is affected and that the irreducible two-body component is not affected by the stripping. Therefore, $\tilde{h}_{\alpha \lambda}^{(2)}$ is the correlation function with a truly irreducible two-body behaviour, not $h_{\alpha \lambda}^{(2)}$. Lastly, as equation (28) shows that $\Gamma_{\alpha \lambda}=\Gamma_{\lambda \alpha}$, the exchange symmetry $\{\alpha, \mathbf{x}\} \leftrightarrow\{\lambda, \mathbf{y}\}$ is clearly preserved. The full truncated correlation function $\tilde{t}^{(2)}$ associated to $\tilde{h}^{(2)}$ is

$$
\begin{aligned}
\tilde{t}_{\alpha \lambda}^{(2)}(\mathbf{x}, \mathbf{y}) & =n_{\alpha}^{(1)}(\mathbf{x}) n_{\lambda}^{(1)}(\mathbf{y}) \tilde{h}_{\alpha \lambda}^{(2)}(\mathbf{x}, \mathbf{y})+n_{\alpha}^{(1)}(\mathbf{x}) \delta_{\alpha \lambda} \delta(\mathbf{x}-\mathbf{y}) \\
& = \begin{cases}t_{\alpha \lambda}^{(2)}(\mathbf{x}, \mathbf{y})-\Gamma_{\alpha \lambda} \frac{\partial n_{\alpha}^{(1)}(\mathbf{x})}{\partial \rho_{\alpha}} \frac{\partial n_{\lambda}^{(1)}(\mathbf{y})}{\partial \rho_{\lambda}} & \mathbf{x} \neq \mathbf{y} \\
t_{\alpha \lambda}^{(2)}(\mathbf{x}, \mathbf{y}) & \mathbf{x}=\mathbf{y}\end{cases}
\end{aligned}
$$


In order to write the unkwnown constant $\Gamma_{\alpha \lambda}$ as a functional of $\tilde{h}_{\alpha \lambda}^{(2)}$ let us first show that $\partial n_{\alpha}^{(1)}(\mathbf{x}) / \partial \rho_{\alpha} \equiv 1$. As $V \rho_{\alpha}=\int n_{\alpha}^{(1)}(\mathbf{x}) \mathrm{d} \mathbf{x}$, then $\partial \rho_{\alpha} / \partial n_{\alpha}^{(1)}(\mathbf{v})=1 / V$ and, so, $\partial n_{\alpha}^{(1)}(\mathbf{x}) / \partial \rho_{\alpha} \equiv 1$. Using this result in equation (18) it is obtained that

$$
\int \tilde{t}_{\alpha \lambda}^{(2)}(\mathbf{x}, \mathbf{y}) \mathrm{d} \mathbf{y d} \mathbf{x}=-\Gamma_{\alpha \lambda} V^{2}
$$

which gets rid of the incompatibility shown in equations (13,18), allows writing the unknown constant $\Gamma_{\alpha \lambda}$ as

$$
\Gamma_{\alpha \lambda}=-\frac{1}{V^{2}} \int n_{\alpha}^{(1)}(\mathbf{x})\left[\delta_{\alpha \lambda}+\int n_{\lambda}^{(1)}(\mathbf{y}) \tilde{h}_{\alpha \lambda}^{(2)}(\mathbf{x}, \mathbf{y}) \mathrm{d} \mathbf{y}\right] \mathrm{d} \mathbf{x}
$$

and to interpret it as a sort of average compressibility. This recovers the classical Lebowitz-Percus results [31] in a form suitable for our purposes. We think that it is quite possible that the factor $\Gamma_{\alpha \lambda}$ provides a formal explanation of the changes observed in properties of confined systems with respect to bulk properties [17, 18, 19, 20], in particular when the system is near critical points and thus account for the observed deviation in the critical point in confined systems [7]. Moreover, it might be possible that the influence of this modification becomes noticeable, even in the thermodynamic limit, when the system is near critical points. It is also interesting to study the normalization of the $N$-modified pair distribution and correlation functions. Writing $h_{\alpha \lambda}^{(2)}=\tilde{h}_{\alpha \lambda}^{(2)}+\delta h_{\alpha \lambda}^{(2)}$ it is obtained that (equations (24), (16) and (17))

$$
\begin{aligned}
& n_{\alpha}^{(1)}(\mathbf{x}) \int n_{\lambda}^{(1)}(\mathbf{y}) \delta h_{\alpha \lambda}^{(2)}(\mathbf{x}, \mathbf{y}) \mathrm{d} \mathbf{y}=\Gamma_{\alpha \lambda} V \\
& n_{\alpha}^{(1)}(\mathbf{x}) \int n_{\lambda}^{(1)}(\mathbf{y}) \tilde{h}_{\alpha \lambda}^{(2)}(\mathbf{x}, \mathbf{y}) \mathrm{d} \mathbf{y}=-n_{\alpha}^{(1)}(\mathbf{x}) \delta_{\alpha \lambda}-\Gamma_{\alpha \lambda} V \\
& \int \tilde{n}_{\alpha \lambda}^{(2)}(\mathbf{x}, \mathbf{y}) \mathrm{d} \mathbf{x} \mathrm{d} \mathbf{y}=N_{\alpha}\left(N_{\lambda}-\delta_{\alpha \lambda}\right)-\Gamma_{\alpha \lambda} V^{2}
\end{aligned}
$$

and, using equation (28) for $\Gamma_{\alpha \lambda}$, it is concluded that equation (31) holds if

$$
\int n_{\alpha}^{(1)}(\mathbf{x}) \mathrm{d} \mathbf{x} \int n_{\lambda}^{(1)}(\mathbf{y}) \mathrm{d} \mathbf{y}=N_{\alpha} N_{\lambda}
$$

regardless of the value of $\int n_{\alpha} n_{\lambda} \tilde{h}_{\alpha \lambda}$. Therefore, the normalization of $n^{(1)}$ is enough to ensure the normalization of $\tilde{n}^{(2)}$ or, in other words, the norm of the $N$-modified pair correlation function is undetermined. This free flotation effectively lifts the fixed $N$ constraint and in the next section it is shown to be the change needed for having a two-body $\mathrm{OZ}$ equation in the $\mathrm{CE}$.

\section{Two-body $\mathrm{OZ}$ equation for the modified kernel}

Now we need to prove that the modified kernel $\tilde{t}_{\alpha \lambda}^{(2)}(\mathbf{x}, \mathbf{y})$ can be inverted. The generalization to higher order functions is done in Sections 5 and 6 . and it is needed not only for having a well defined $N$-modified distribution functions hierarchy, but also 
for formulating a well defined CE set of OZ equations that operate on arbitrary $n$-body configurations. If the fields are arbitrarily varied, we get, from equations (12) and (26)

$$
\delta n_{\alpha}^{(1)}(\mathbf{x})=\sum_{\lambda} \int \tilde{t}_{\alpha \lambda}^{(2)}(\mathbf{x}, \mathbf{y}) \delta \Phi_{\lambda}(\mathbf{y}) \mathrm{d} \mathbf{y}+\sum_{\lambda} \Gamma_{\alpha \lambda} \frac{\partial}{\partial \rho_{\lambda}} \int n_{\lambda}^{(1)}(\mathbf{y}) \delta \Phi_{\lambda}(\mathbf{y}) \mathrm{d} \mathbf{y}
$$

But equation (田) shows that, when only $\Phi_{\lambda}$ varies

$$
\delta \ln Q=-\beta \delta F=\int n_{\lambda}^{(1)}(\mathbf{y}) \delta \Phi_{\lambda}(\mathbf{y}) \mathrm{d} \mathbf{y}
$$

and, as the partial chemical potential for $\lambda$ particles is $\left.\mu_{\lambda}=V^{-1} \partial F / \partial \rho_{\lambda}\right)_{V, T}$, then

$$
\int n_{\lambda}^{(1)}(\mathbf{y}) \delta \Phi_{\lambda}(\mathbf{y}) \mathrm{d} \mathbf{y}=-\beta V \delta \mu_{\lambda}
$$

we can write

$$
\delta n_{\alpha}^{(1)}(\mathbf{x})=\sum_{\lambda} \int \tilde{t}_{\alpha \lambda}^{(2)}(\mathbf{x}, \mathbf{y}) \delta \Phi_{\lambda}(\mathbf{y}) \mathrm{d} \mathbf{y}-\sum_{\lambda} \Gamma_{\alpha \lambda} \beta V \delta \mu_{\lambda}
$$

and using equation (27) it is obtained that

$$
\begin{aligned}
\delta n_{\alpha}^{(1)}(\mathbf{x}) & =\sum_{\lambda} \int \tilde{t}_{\alpha \lambda}^{(2)}(\mathbf{x}, \mathbf{y}) \delta \Phi_{\lambda}(\mathbf{y}) \mathrm{d} \mathbf{y}+\sum_{\lambda} \int \tilde{t}_{\alpha \lambda}^{(2)}(\mathbf{x}, \mathbf{y}) \beta \delta \mu_{\lambda} \mathrm{d} \mathbf{y} \\
& =\sum_{\lambda} \int \tilde{t}_{\alpha \lambda}^{(2)}(\mathbf{x}, \mathbf{y}) \delta \tilde{\Phi}_{\lambda}(\mathbf{y}) \mathrm{d} \mathbf{y}
\end{aligned}
$$

where $\tilde{\Phi}_{\lambda}(\mathbf{y})$ is

$$
\tilde{\Phi}_{\lambda}(\mathbf{y})=\Phi_{\lambda}(\mathbf{y})+\beta \mu_{\lambda}
$$

Therefore, an inverse modified kernel $\tilde{t}_{\eta \alpha}^{(2)-1}(\mathbf{z}, \mathbf{x})$ exists and complies with equation (13) if the modified field $\tilde{\Phi}$ is used instead of $\Phi$. Writing

$$
\tilde{t}_{\eta \alpha}^{(2)-1}(\mathbf{z}, \mathbf{x})=\frac{\delta \tilde{\Phi}_{\eta}(\mathbf{z})}{\delta n_{\alpha}^{(1)}(\mathbf{x})}=-\tilde{c}_{\eta \alpha}^{(2)}(\mathbf{z}, \mathbf{x})+\frac{\delta_{\eta \alpha} \delta(\mathbf{z}-\mathbf{x})}{n_{\alpha}^{(1)}(\mathbf{x})}
$$

(which defines the $N$-modified direct correlation function $\tilde{c}_{\eta \alpha}^{(2)}$ ) equation (13) becomes the $\mathrm{OZ}$ equation in the $\mathrm{CE}$ where the $N$-modified pair correlation functions $\tilde{h}_{\eta \lambda}^{(2)}$ and $\tilde{c}_{\eta \lambda}^{(2)}$ must be used

$$
\tilde{h}_{\eta \lambda}^{(2)}(\mathbf{x}, \mathbf{y})-\tilde{c}_{\eta \lambda}^{(2)}(\mathbf{x}, \mathbf{y})=\sum_{\alpha} \int n_{\alpha}^{(1)}(\mathbf{z}) \tilde{c}_{\eta \alpha}^{(2)}(\mathbf{x}, \mathbf{z}) \tilde{h}_{\alpha \lambda}^{(2)}(\mathbf{z}, \mathbf{y}) \mathrm{d} \mathbf{z}
$$

So, up to now, we have seen that the constant $N$ constraint induces a long range behavior in the conventional pair correlation function incompatible with being an irreducible two-body function and have also shown that, if this behaviour (expressed in the separation of variables of equation (23) $)$ is dropped off when defining the $N$-modified pair correlation functions (thus getting a truly irreducible two-body function), then an 
authentic OZ equation for the $N$-modified pair correlation functions is obtained. This generalizes Ramshaw's results to pair correlations in a mixture. Let us recall that it is essential to show that this scheme gives a coherent view of all the possible $n$-body configurations in a liquid mixture, i.e., it is not limited to pair functions. This is done in the next two sections.

\section{The $N$-modified canonical hierarchy}

For the sake of clarity we limit ourselves, in this section, to prove that we really have another hierarchy of distribution and correlation functions modified in such a way that the fixed $N$ constraint is properly taken into account and, in the next section the new hierarchy is used to prove the extension of the HKSM theorem to the CE.

We first explicitly write down the modified canonical distribution function $\tilde{h}$ for more than two bodies and perform the same sort of analysis as done in Sections 2 and 3 and then turn to the functional analysis techniques in order to show that, by deriving

with respect to $\tilde{\Phi}$, we can generate higher order distribution functions. In this way we not only show that functional analysis techniques are simpler but also obtain, from the first method, some additional physical insight. As usual, $\tilde{h}^{(3)}$ will be enough. First of all, the definition and normalization equations for the three body functions are

$$
n_{\alpha \lambda \eta}^{(3)}(\mathbf{x}, \mathbf{y}, \mathbf{z})= \begin{cases}\left\langle\sum_{k_{\alpha}} \delta\left(\mathbf{x}-\mathbf{r}_{k_{\alpha}}\right) \sum_{l_{\alpha}} \delta\left(\mathbf{y}-\mathbf{r}_{l_{\alpha}}\right) \sum_{m_{\alpha}} \delta\left(\mathbf{z}-\mathbf{r}_{m_{\alpha}}\right)\right\rangle & \alpha \neq \lambda \neq \eta \\ \left\langle\sum_{k_{\alpha} \neq l_{\alpha}} \delta\left(\mathbf{x}-\mathbf{r}_{k_{\alpha}}\right) \sum_{l_{\alpha}} \delta\left(\mathbf{y}-\mathbf{r}_{l_{\alpha}}\right) \sum_{m_{\eta}} \delta\left(\mathbf{z}-\mathbf{r}_{m_{\eta}}\right)\right\rangle & \alpha=\lambda \neq \eta \\ \left\langle\sum_{k_{\alpha} \neq m_{\alpha}} \delta\left(\mathbf{x}-\mathbf{r}_{k_{\alpha}}\right) \sum_{l_{\lambda}} \delta\left(\mathbf{y}-\mathbf{r}_{l_{\lambda}}\right) \sum_{m_{\alpha}} \delta\left(\mathbf{z}-\mathbf{r}_{m_{\alpha}}\right)\right\rangle & \alpha=\eta \neq \lambda \\ \left\langle\sum_{k_{\alpha}} \delta\left(\mathbf{x}-\mathbf{r}_{k_{\alpha}}\right) \sum_{l_{\lambda} \neq m_{\lambda}} \delta\left(\mathbf{y}-\mathbf{r}_{l_{\lambda}}\right) \sum_{m_{\lambda}} \delta\left(\mathbf{z}-\mathbf{r}_{m_{\lambda}}\right)\right\rangle & \alpha \neq \lambda=\eta \\ \left\langle\sum_{k_{\alpha} \neq l_{\alpha} \neq m_{\alpha}} \delta\left(\mathbf{x}-\mathbf{r}_{k_{\alpha}}\right) \sum_{l_{\alpha} \neq m_{\alpha}} \delta\left(\mathbf{y}-\mathbf{r}_{l_{\lambda}}\right) \sum_{m_{\alpha}} \delta\left(\mathbf{z}-\mathbf{r}_{m_{\eta}}\right)\right\rangle & \alpha=\lambda=\eta\end{cases}
$$

The normalization equations are, in the $\mathrm{CE}$

$$
\begin{aligned}
& \int n_{\alpha \lambda \eta}^{(3)}(\mathbf{x}, \mathbf{y}, \mathbf{z}) \mathrm{d} \mathbf{x} \mathrm{d} \mathbf{y} \mathrm{d} \mathbf{z}=N_{\alpha}\left(N_{\lambda}-\delta_{\alpha \lambda}\right)\left(N_{\eta}-\delta_{\alpha \eta}-\delta_{\lambda \eta}\right) \\
& \int n_{\eta}^{(1)}(\mathbf{z}) h_{\alpha \lambda \eta}^{(3)}(\mathbf{x}, \mathbf{y}, \mathbf{z}) \mathrm{d} \mathbf{z}=-\left(\delta_{\alpha \eta}+\delta_{\lambda \eta}\right) h_{\alpha \lambda}^{(2)}(\mathbf{x}, \mathbf{y}) \\
& \int n_{\lambda}^{(1)}(\mathbf{y}) n_{\eta}^{(1)}(\mathbf{z}) h_{\alpha \lambda \eta}^{(3)}(\mathbf{x}, \mathbf{y}, \mathbf{z}) \mathrm{d} \mathbf{y} \mathrm{d} \mathbf{z}=\delta_{\alpha \lambda}\left(\delta_{\alpha \eta}+\delta_{\lambda \eta}\right) \\
& \int n_{\alpha}^{(1)}(\mathbf{x}) n_{\lambda}^{(1)}(\mathbf{y}) n_{\eta}^{(1)}(\mathbf{z}) h_{\alpha \lambda \eta}^{(3)}(\mathbf{x}, \mathbf{y}, \mathbf{z}) \mathrm{d} \mathbf{x} \mathrm{d} \mathbf{y} \mathrm{d} \mathbf{z}=N_{\alpha} \delta_{\alpha \lambda}\left(\delta_{\alpha \eta}+\delta_{\lambda \eta}\right)
\end{aligned}
$$


We can write the $N$-modified canonical correlation function $\tilde{h}^{(3)}$ as

$$
h_{\alpha \lambda \eta}^{(3)}(\mathbf{x}, \mathbf{y}, \mathbf{z})=\tilde{h}_{\alpha \lambda \eta}^{(3)}(\mathbf{x}, \mathbf{y}, \mathbf{z})+\delta h_{\alpha \lambda \eta}^{(3)}(\mathbf{x}, \mathbf{y}, \mathbf{z})
$$

The interesting point to emphasize here is that, as Lebowitz and Percus [32] have already discussed, the long range behaviour of an $n$-body function in the CE ensemble depends on how the clustering of the $n$ molecules is. Specifically, and in our case $(n=3$, long range behaviour), we can have the three molecules very far away from each other as well as a 2-molecules cluster and the other one very far of them. It is clear that the complexity of the accounting for all the clustering possibilities increases exponentially with $n$ and that, in principle, one has to define different constants in equations like (29) and (44), one for each kind of clustering. This is more than enough justification to follow up the functional differentiation approach instead of the one summarized above. However, it is also instructive to show that, if we consider the case of the three molecules, every one of them very far from each other, then it is obtained, by using equation (18), that

$$
\begin{aligned}
\int n_{\alpha}^{(1)}(\mathbf{x}) n_{\lambda}^{(1)}(\mathbf{y}) n_{\eta}^{(1)}(\mathbf{z}) \delta \tilde{h}_{\alpha \lambda \eta}^{(3)}(\mathbf{x}, \mathbf{y}, \mathbf{z}) \mathrm{d} \mathbf{x} \mathrm{d} \mathbf{y} \mathrm{d} \mathbf{z}= & \Gamma_{\alpha \lambda \eta} V^{3}\left[1-\frac{\rho_{\alpha} \delta_{\alpha \lambda}}{V}-\frac{\rho_{\lambda} \delta_{\lambda \eta}}{V}-\right. \\
& \left.\frac{\rho_{\eta} \delta_{\alpha \eta}}{V}+\frac{\rho_{\alpha} \delta_{\lambda \eta}}{V^{2}}+\frac{\rho_{\lambda} \delta_{\alpha \eta}}{V^{2}}+\frac{\rho_{\eta} \delta_{\alpha \lambda}}{V^{2}}\right]
\end{aligned}
$$

If we can take the thermodynamic limit, the constant $\Gamma_{\alpha \lambda \eta}$ is, using equation (42),

$\Gamma_{\alpha \lambda \eta}=\frac{1}{V^{3}} \int n_{\alpha}^{(1)}(\mathbf{x})\left[\delta_{\alpha \lambda}\left(\delta_{\alpha \eta}+\delta_{\lambda \eta}\right)-\int n_{\lambda}^{(1)}(\mathbf{y}) n_{\eta}^{(1)}(\mathbf{z}) \tilde{h}_{\alpha \lambda \eta}^{(3)}(\mathbf{x}, \mathbf{y}, \mathbf{z}) \mathrm{d} \mathbf{y} \mathrm{d} \mathbf{z}\right] \mathrm{d} \mathbf{x}$

This shows that, if the size of the system allows this kind of limit, the long range behaviour of $n$-body configurations is (in the CE) described by (up to) $n$ order susceptibilities. Lastly, let us also mention that, working along the same lines that led to equation (32), it is concluded that the norm of $h^{(3)}$ is also undetermined.

In the functional point of view, the argument is as follows: if the densities and fields can be thought of as equivalent sets of independent variables, i.e. given $\left\{n_{\alpha}\right\}$ the fields $\left\{\Phi_{\lambda}\right\}$ are a unique functional of them and viceversa, then each one of them can be written as a functional Taylor series in the form

$$
\begin{aligned}
& \delta n_{\alpha}^{(1)}(\mathbf{x})=\sum_{\lambda} \int \frac{\delta n_{\alpha}^{(1)}(\mathbf{x})}{\delta \Phi_{\lambda}(\mathbf{y})} \delta \Phi_{\lambda}(\mathbf{y}) \mathrm{d} \mathbf{y}+\frac{1}{2} \sum_{\lambda, \eta} \int \frac{\delta^{2} n_{\alpha}^{(1)}(\mathbf{x})}{\delta \Phi_{\lambda}(\mathbf{y}) \delta \Phi_{\eta}(\mathbf{z})} \delta \Phi_{\lambda}(\mathbf{y}) \delta \Phi_{\eta}(\mathbf{z}) \mathrm{d} \mathbf{y} \mathrm{d} \mathbf{z}+\ldots(46) \\
& \delta \Phi_{\alpha}(\mathbf{x})=\sum_{\lambda} \int \frac{\delta \Phi_{\alpha}(\mathbf{x})}{\delta n_{\lambda}^{(1)}(\mathbf{y})} \delta n_{\lambda}^{(1)}(\mathbf{y}) \mathrm{d} \mathbf{y}+\frac{1}{2} \sum_{\lambda, \eta} \int \frac{\delta^{2} \Phi_{\alpha}(\mathbf{x})}{\delta n_{\lambda}^{(1)}(\mathbf{y}) \delta n_{\eta}^{(1)}(\mathbf{z})} \delta n_{\lambda}^{(1)}(\mathbf{y}) \delta n_{\eta}^{(1)}(\mathbf{z}) \mathrm{d} \mathbf{y} \mathrm{d} \mathbf{z}+\ldots
\end{aligned}
$$

Substituting $\delta \Phi$ in equation (46) and equating powers of $\delta n$, it is obtained, up to second order, that

$$
\delta_{\alpha \omega} \delta(\mathbf{x}-\mathbf{u})=\sum_{\lambda} \int \frac{\delta n_{\alpha}^{(1)}(\mathbf{x})}{\delta \Phi_{\lambda}(\mathbf{y})} \frac{\delta \Phi_{\lambda}(\mathbf{y})}{\delta n_{\omega}^{(1)}(\mathbf{u})} \mathrm{d} \mathbf{y}
$$


$0=\sum_{\lambda} \int \frac{\delta n_{\alpha}^{(1)}(\mathbf{x})}{\delta \Phi_{\lambda}(\mathbf{y})} \frac{\delta^{2} \Phi_{\lambda}(\mathbf{y})}{\delta n_{\omega}^{(1)}(\mathbf{u}) \delta n_{\nu}^{(1)}(\mathbf{v})} \mathrm{d} \mathbf{y}+\sum_{\lambda, \eta} \int \frac{\delta^{2} n_{\alpha}^{(1)}(\mathbf{x})}{\delta \Phi_{\lambda}(\mathbf{y}) \delta \Phi_{\eta}(\mathbf{z})} \frac{\delta \Phi_{\lambda}(\mathbf{y})}{\delta n_{\omega}^{(1)}(\mathbf{u})} \frac{\delta \Phi_{\eta}(\mathbf{z})}{\delta n_{\nu}^{(1)}(\mathbf{v})} \mathrm{d} \mathbf{y} \mathrm{d} \mathbf{z}(49)$

Let us first analyze these equations in the GCE, i.e. both equations (46,47) are certainly correct. In that case, equation (48) just leads to the OZ equation. Also, as equation (49) holds for all $\{\alpha, \mathbf{x}\},\{\omega, \mathbf{u}\},\{\nu, \mathbf{v}\}$ values and, if we use that (see equations $(8,37))$

$$
\begin{aligned}
\hat{t}_{\alpha \lambda \eta}^{(3)}(\mathbf{x}, \mathbf{y}, \mathbf{z}) & =\frac{\delta^{2} n_{\alpha}^{(1)}(\mathbf{x})}{\delta \Phi_{\lambda}(\mathbf{y}) \delta \Phi_{\eta}(\mathbf{z})} \\
\hat{t}_{\alpha \lambda \eta}^{(3)-1}(\mathbf{x}, \mathbf{y}, \mathbf{z}) & =\frac{\delta^{2} \Phi_{\alpha}(\mathbf{x})}{\delta n_{\lambda}^{(1)}(\mathbf{y}) \delta n_{\eta}^{(1)}(\mathbf{z})}=\frac{\delta}{\delta n_{\lambda}^{(1)}(\mathbf{y})}\left[\hat{t}_{\alpha \eta}^{(2)-1}(\mathbf{x}, \mathbf{z})\right]= \\
& =-\frac{\delta_{\alpha \lambda} \delta_{\alpha \eta} \delta(\mathbf{x}-\mathbf{y}) \delta(\mathbf{x}-\mathbf{z})}{\left(n_{\alpha}^{(1)}(\mathbf{x})\right)^{2}}-c_{\alpha \lambda \eta}^{(3)}(\mathbf{x}, \mathbf{y}, \mathbf{z})
\end{aligned}
$$

in equation (49), multiply by $\hat{t}_{\sigma \alpha}^{(2)-1}(\mathbf{s}, \mathbf{x})$, operate with $\sum_{\alpha} \int d \mathbf{x}$ and use equation (48), the result is

$\hat{t}_{\sigma \omega \nu}^{(3)-1}(\mathbf{s}, \mathbf{u}, \mathbf{v})=-\sum_{\alpha, \lambda, \eta} \int \hat{t}_{\sigma \alpha}^{(2)-1}(\mathbf{s}, \mathbf{x}) \hat{t}_{\alpha \lambda \eta}^{(3)}(\mathbf{x}, \mathbf{y}, \mathbf{z}) \hat{t}_{\lambda \omega}^{(2)-1}(\mathbf{y}, \mathbf{u}) \hat{t}_{\eta \nu}^{(2)-1}(\mathbf{z}, \mathbf{v}) \mathrm{d} \mathbf{x} \mathrm{d} \mathbf{y} \mathrm{d} \mathbf{z}$

This is the OZ equation for the three-body configurations and the procedure shows how to write $\mathrm{OZ}$ equations for $n$-body configurations, i.e. if the first $p$-body OZ equations are needed, a Taylor expansion of both $\left\{n_{\alpha}^{(1)}\right\}$ and $\left\{\tilde{\Phi}_{\lambda}\right\}$ is performed, one series is substituted into the other and terms up to order $p-1$ are equated. These equations have been discussed before, see e.g. [33], 34, 35], and we have followed the more straightforward Henderson's method. Notice also that $c_{\alpha \lambda \eta}^{(3)}$ has been generated by density differentiation of $c_{\alpha \lambda}^{(2)}$. Therefore, $\hat{t}^{(3)-1}$ exists if and only if all the $\hat{t}^{(2)-1}$ exist. Let us now turn to the CE. If equations (46,47) make sense in the CE, then in equations $(48,49)$ the derivatives must be taken w.r.t. $\tilde{\Phi}$ and, as a consequence, $\hat{t}$ is replaced by $\tilde{t}$ everywhere and equation (52) (with $\tilde{t}$ instead of $\hat{t}$ ) is the three-body OZ equation for the $N$-modified correlation functions. Also, equation (51) shows that, as is customary, the $N$-modified direct correlation hierarchy is built by deriving with respect to the local density. As $\hat{t}^{(n)-1}$ depends, to all orders, on the lower order derivatives $\hat{t}^{(k)-1}, k<n$, we conclude that the operators $\delta / \delta \tilde{\Phi}$ and $\delta / \delta n$ generate the hierarchies of $N$-modified canonical total and direct correlation functions $\tilde{t}$ and $\tilde{c}$ respectively, with $\hat{t}$ replaced by $\tilde{t}$ and $\Phi$ by $\tilde{\Phi}$ everywhere, that the usual formal links between both correlation functions are kept and, lastly, let us mention that the missing link of how the direct correlation hierarchy starts is discussed in the next section (see equation (65)) where we identify the first member of the $N$-modified direct correlation with the density derivative of our $N$-modified free energy functional. 


\section{HKSM theorem in the CE}

Here we propose an $N$-modified free energy and thermodynamic potential functional, show that the latter is minimized by the equilibrium density profiles and identify the first members of the $N$-modified hierarchies completing in that way the proof that we really have a new distribution functions hierarchy with its associated truncated and direct correlation functions hierarchies and that the HKSM theorem can be extended to the CE.

First of all, let us summarize the standard formulation of DFT in the GCE. It starts from the HKSM theorem which states that, in the GCE, not only the external potential unequivocally determines the density profile, it is also true that the density profile unequivocally determines an external potential [13]. Moreover, the grand thermodynamic potential functional is a minimum for the equilibrium density profile. This result allows to write it as (following 四 in our notation)

$$
\beta \Omega\left[\left\{n^{(1)}\right\}\right]=\beta F\left[\left\{n^{(1)}\right\}\right]-\sum_{\alpha} \int\left[\Phi_{\alpha}(\mathbf{x})+\beta \mu_{\alpha}\right] n_{\alpha}^{(1)}(\mathbf{x}) \mathrm{d} \mathbf{x}
$$

$F$ is the free energy functional of the system

$$
\beta F\left[\left\{n^{(1)}\right\}\right]=\left\langle\beta\left(K_{N}+U_{N}-\ln P_{N}\right)\right\rangle
$$

$K_{N}$ is the kinetic energy, $U_{N}$ the interaction energy, $P_{N}$ the grand canonical probability density and the average is a grand canonical one. Let us emphasize that the entropic term $\ln P_{N}$ depends on all the $n$-body configurations and, therefore, is responsible for the need of a full hierarchy of distribution functions. The variational principle that determines the density profile is obtained from

$$
\frac{\delta \beta \Omega\left[\left\{n^{(1)}\right\}\right]}{\delta n_{\alpha}^{(1)}(\mathbf{x})}=0
$$

Defining the intrinsic chemical potential by

$$
\mu_{\alpha}^{(i n t)}\left[\left\{n^{(1)}\right\} ; \mathbf{x}\right]=\frac{\delta F\left[\left\{n^{(1)}\right\}\right]}{\delta n_{\alpha}^{(1)}(\mathbf{x})}
$$

equation (55) becomes

$$
\beta \mu_{\alpha}^{(i n t)}\left[\left\{n^{(1)}\right\} ; \mathbf{x}\right]=\Phi_{\alpha}(\mathbf{x})+\beta \mu_{\alpha}=\hat{\Phi}_{\alpha}(\mathbf{x})
$$

where $\mu_{\alpha}$ is the chemical potential and, separating the ideal and excess contributions to the free energy $F=F^{(i d)}-F^{(e x c)}$

$$
\begin{aligned}
& \beta F^{(i d)}=\sum_{\alpha} \int n_{\alpha}^{(1)}(\mathbf{x})\left[\ln \left(\lambda^{3} n_{\alpha}^{(1)}(\mathbf{x})\right)-1\right] \mathrm{d} \mathbf{x} \\
& \beta \mu_{\alpha}^{(i d)}=\ln \left(\lambda^{3} n_{\alpha}^{(1)}(\mathbf{x})\right)
\end{aligned}
$$


$\lambda$ is the thermal wavelength and the one body direct correlation function is defined by

$$
c_{\alpha}^{(1)}(\mathbf{x})=\beta \frac{\delta F^{(e x c)}}{\delta n_{\alpha}^{(1)}(\mathbf{x})}=\ln \left(\lambda^{3} n_{\alpha}^{(1)}(\mathbf{x})\right)-\hat{\Phi}_{\alpha}(\mathbf{x})
$$

which is the usual equation for the density profile 4 . The next direct correlation function is

$$
c_{\alpha \lambda}^{(2)}(\mathbf{x}, \mathbf{y})=\frac{\delta c_{\alpha}^{(1)}(\mathbf{x})}{\delta n_{\lambda}^{(1)}(\mathbf{y})}=\frac{\delta_{\alpha \lambda} \delta(\mathbf{x}-\mathbf{y})}{n_{\alpha}^{(1)}(\mathbf{x})}-\frac{\delta \hat{\Phi}_{\alpha}^{(1)}(\mathbf{x})}{\delta n_{\lambda}^{(1)}(\mathbf{y})}
$$

In this way the distribution function hierarchies are generated and a density functional theory in the GCE is established.

Now let us turn to the CE formulation. Equations (13) and (18) show that a straightforward formulation is doomed: the inverse kernel does not exist. In order to complete the extension of the HKSM theorem to the CE let us write an $N$-modified free energy functional, show that there exists a variational principle in the density profile and that a whole set of $N$-modifed distribution function hierarchies is generated by this functional. Our proposal is

$$
\beta \mathcal{F}\left[\left\{\tilde{n}^{(1)}\right\}\right]=\left\langle\beta\left(K_{N}+U_{N}-\ln P_{N}\right)\right\rangle
$$

where now both $P_{N}$ and the average are canonical and the $N$-modified distribution functions $\tilde{n}$ are used. The $N$-modified grand potential functional to minimize is

$$
\beta \tilde{\Omega}\left[\left\{\tilde{n}^{(1)}\right\}\right]=\beta \mathcal{F}\left[\left\{\tilde{n}^{(1)}\right\}\right]-\sum_{\alpha} \int \tilde{n}_{\alpha}^{(1)}(\mathbf{x}) \tilde{\Phi}_{\alpha}(\mathbf{x}) \mathrm{d} \mathbf{x}
$$

Let us first show that $\tilde{\Omega}$ is minimum when the equilibrium density profiles are used in the average of equation (63);

$\beta \tilde{\Omega}=\left\langle\beta\left(H-\sum_{\alpha} \mu_{\alpha}-\ln P_{N}\right)\right\rangle=\int P_{N}(\{\mathbf{x}\})\left[\beta\left(H-\sum_{\alpha} \mu_{\alpha}-\ln P_{N}\right)\right] \mathrm{d}\{\mathbf{x}\}$

and, with any other probability distribution $P_{N}^{\prime}(\{\mathbf{x}\})$ such that

$$
\int P_{N}^{\prime}(\{\mathbf{x}\}) d\{\mathbf{x}\}=\int P_{N}(\{\mathbf{x}\}) \mathrm{d}\{\mathbf{x}\}
$$

the Gibbs-Bogoliubov inequality [30] states that

$$
\beta \Omega^{\prime}=\int P_{N}^{\prime}(\{\mathbf{x}\})\left[\beta\left(H-\sum_{\alpha} \mu_{\alpha}-\ln P_{N}\right)\right] \mathrm{d}\{\mathbf{x}\} \geq \beta \tilde{\Omega}
$$

So, the variational equations are, separating as in the grand canonical case the ideal and excess contributions,

$$
\tilde{c}_{\alpha}^{(1)}(\mathbf{x})=\beta \frac{\delta \mathcal{F}^{(e x c)}}{\delta \tilde{n}_{\alpha}^{(1)}(\mathbf{x})}=\ln \left(\lambda^{3} \tilde{n}_{\alpha}^{(1)}(\mathbf{x})\right)-\tilde{\Phi}_{\alpha}(\mathbf{x})
$$


This equation for the density profile has the same form as in equation (60). The two body direct correlation function is

$$
\tilde{c}_{\alpha \lambda}^{(2)}(\mathbf{x}, \mathbf{y})=\frac{\delta \tilde{c}_{\alpha}^{(1)}(\mathbf{x})}{\delta \tilde{n}_{\lambda}^{(1)}(\mathbf{y})}=\frac{\delta_{\alpha \lambda} \delta(\mathbf{x}-\mathbf{y})}{\tilde{n}_{\alpha}^{(1)}(\mathbf{x})}-\frac{\delta \tilde{\Phi}_{\alpha}^{(1)}(\mathbf{x})}{\delta \tilde{n}_{\lambda}^{(1)}(\mathbf{y})}
$$

and it has the same form as equation (61). Therefore, it is clearly seen that for the $N$-modified thermodynamic potential defined in equation (63) a HKSM theorem can be formulated and a DFT for the CE is established. Moreover, the thermodynamic potential of equation (63) shows that the $N$-modified formulation of the distribution functions allows us to work in a $\mathrm{CE}$ as if it were a grand canonical one. Also, if the system's size forces us to explicitly consider size effects, then equations like $(26,28)$ contain a recipe to include size effects. A point worth of mention is that in the first sections of this paper we wrote the density profile as $n_{\alpha}^{(1)}$ and now, in this section, we have used $\tilde{n}_{\alpha}^{(1)}$. In fact, both are the same $\left(n_{\alpha}^{(1)}=\tilde{n}_{\alpha}^{(1)}=t_{\alpha}^{(1)}=\ldots\right)$ but, as the CE free energy and the $N$-modified free energy also depend on $n_{\alpha \lambda}^{(2)}$ and $\tilde{n}_{\alpha \lambda}^{(2)}$ respectively, they are not the same functionals and so, the hierarchies generated by functional differentiation are different. Therefore, we use $\tilde{n}_{\alpha}^{(1)}$ just for the sake of notational consistency.

\section{Conclusions}

Summarizing, we have proved that, if the long range behaviour of the CE distribution and correlation functions is, roughly speaking, left aside, the HKSM theorem and the OZ equation can be extended to the $\mathrm{CE}$. As the dismissal of the long range behaviour can be interpreted as taking an open set within the $\mathrm{CE}$ (the textbook procedure to obtain the GCE) this seems to be an eminently logical result. We have also generated, first by direct construction and then by functional differentiation, a new set of $N$-modified distribution and correlation functions that obey the same rules and have the same formal structure that the more conventional ones have. The point here is that the chemical potential modifies the external field and these new functions have the correct $n$-body irreducible behaviour. It can be mentioned that a price to pay for that is to give up the normalization equations for the more than one-body correlation functions. In fact, only the density profile is normalized to the total number of particles and the other functions freely float. The direct construction method also shows that the long range terms dropped off depend on $n$-body susceptibilities. We have also shown that the functional differentiation procedure is the amenable one when seeking a general theory but the direct construction method shows the influence of the $n$-body compressibilities and is, in this sense, physically enlightening. It might even be thought that its influence could be felt, even in macroscopic systems, near the critical points but more specifric work is

needed. These $N$-modified functions $\tilde{n}_{\alpha_{1} \ldots \alpha_{s}}^{(s)}\left(\mathbf{x}_{1} \ldots \mathbf{x}_{s}\right), \tilde{t}_{\alpha_{1} \ldots \alpha_{s}}^{(s)}\left(\mathbf{x}_{1} \ldots \mathbf{x}_{s}\right), \tilde{c}_{\alpha_{1} \ldots \alpha_{s}}^{(s)}\left(\mathbf{x}_{1} \ldots \mathbf{x}_{s}\right)$ are defined by

$$
\tilde{n}_{\alpha_{1} \ldots \alpha_{s}}^{(s)}\left(\mathbf{x}_{1} \ldots \mathbf{x}_{s}\right)=\frac{1}{Q} \frac{\delta^{s} Q}{\prod_{k=1}^{s} \delta \tilde{\Phi}_{\alpha_{s} k}\left(\mathbf{x}_{k}\right)}
$$




$$
\begin{aligned}
& \tilde{t}_{\alpha_{1} \ldots \alpha_{s}}^{(s)}\left(\mathbf{x}_{1} \ldots \mathbf{x}_{s}\right)=\frac{\delta^{s} \ln Q}{\prod_{k=1}^{s} \delta \tilde{\Phi}_{\alpha_{s} k}\left(\mathbf{x}_{k}\right)}=\frac{\delta \tilde{t}_{\alpha_{1} \ldots \alpha_{s-1}}^{(s-1)}\left(\mathbf{x}_{1} \ldots \mathbf{x}_{s-1}\right)}{\delta \tilde{\Phi}_{\alpha_{s}}\left(\mathbf{x}_{s}\right)} \\
& \tilde{c}_{\alpha_{1} \ldots \alpha_{s}}^{(s)}\left(\mathbf{x}_{1} \ldots \mathbf{x}_{s}\right)=\beta \frac{\delta^{s} F^{(e x c)}}{\prod_{k=1}^{s} \delta \tilde{n}_{\alpha_{k}}^{(1)}\left(\mathbf{x}_{k}\right)}=\frac{\delta \tilde{c}_{\alpha_{1} \ldots \alpha_{s-1}}^{(s-1)}\left(\mathbf{x}_{1} \ldots \mathbf{x}_{s-1}\right)}{\delta \tilde{n}_{\alpha_{s}}^{(1)}\left(\mathbf{x}_{s}\right)}
\end{aligned}
$$

These equations have the same structure as equations (5),(8) and (9), while the direct correlation hierarchy is initiated by

$$
\tilde{c}_{\alpha}^{(1)}(\mathbf{x})=\ln \left[\lambda^{3} \tilde{n}_{\alpha}^{(1)}(\mathbf{x})\right]-\tilde{\Phi}_{\alpha}(\mathbf{x})
$$

Lastly, let us also mention that, although in the introduction we have emphasized the possible use of these results on mesoscopic systems, the next paper in this series will deal with a system (lattice gas) that, interestingly enough, needs of these results even in the thermodynamic limit.

\section{Acknowledgments}

Extremely helpful and enlightening discussions with Prof. L. Blum, which gave rise to this work, are gratefully acknowledged. We also acknowledge support from Fundacion José A. Balseiro and from CONICET through grant PIP 0859/98.

\section{References}

[1] Hohenberg P and Kohn W 1964 Phys. Rev. 136 B864; Kohn W and Sham L J 1965 Phys. Rev. 140 A1133

[2] Mermin N D 1965 Phys. Rev. 137 A1441

[3] See, e.g., the reviews by Haymet A D J 1987 Annu. Rev. Phys. Chem. 38 89; Evans R 1992 Fundamentals of Inhomogeneous Fluids ed. D. Henderson (New York: M. Dekker); Löwen H 1994 Phys. Rep. 237249 ; Sweatman M B 2000 Mol. Phys. 98573

[4] Evans R 1979 Adv. Phys. 28143

[5] Kierlik E and Rosinberg M L 1990 Phys. Rev. A 42 3382; Kierlik E and Rosinberg M L 1991 Phys. Rev A 44 5025; Schmidt M 2000 Phys. Rev. E 624976

[6] Rosenfeld Y 1989 Phys. Rev. Lett. 63 980; for up to date reviews, see Tarazona P and Rosenfeld Y 1999 New approaches to problems in liquid state theory ed. Caccamo C, Hansen J P and Stell G (Dordrecht: Kluwer), p. 293 and Rosenfeld Y ibid., p.303

[7] Huerta A, Pizio O and Sokolowski S 2000 J. Chem. Phys. 112 4286; Segura C J, Vakarin E V, Chapman W G and Holovko M F 1998 J. Chem. Phys. 108 4837; Klapp S and Fortsmann F 1999 Phys. Rev. E 60 3183; Das D, Senapati S and Chandra A 1999 J. Chem. Phys. 1108129

[8] Millan Malo B, Pizio O. Patrykiejew A and Sokolowski S 2001 J. Phys. Cond. Matter 131361 ; Ravikovitch P I, Vishnyakov A and Neimark A V 2001 Phys. Rev. E 64 011602; Bryk P, Patrykiejew A, Reszko-Zygmunt J, Rzysko W and Sokolowski S 1999 Mol. Phys. 96 1509; Yoon T and Kim S 1998 Phys. Rev. E 584541

[9] Gonzalez A, White J A, Roman F L, Velasco S and Evans R 1997 Phys. Rev. Lett. 79 2466; Gonzalez A, White J A, Roman F L and Evans R 1998 J. Chem. Phys. 109 3637; Evans R 1999 New approaches to problems in liquid state theory ed. Caccamo C, Hansen J P and Stell G (Dordrecht: Kluwer) 153.

[10] For an up to date review see Christenson H K 2001 J. Phys. Cond. Matter 13 R95; Morishige K and Ogisu Y 2001 J. Chem. Phys. 114 7166; Tarazona P 2000 Phys. Rev. Lett. 84 694; Klapp S H L and Patey G N 2000 J. Chem. Phys. 11210949 
[11] Xu H and Baus M 2000 Phys. Rev. E 61 3249; Wadewitz T and Winkelmann J 2000 J. Chem. Phys. 1132447

[12] See, e.g., the reviews by Parr R G and Yang W 1989 Density functional Theory of Atoms and Molecules (Oxford: Oxford University Press); Parr R G and Yang W 1995 Annu. Rev. Phys. Chem. 46 710; Kohn W, Becke A D and Parr R G 1996 J. Phys. Chem. 10012974

[13] Chayes J T and Chayes L 1984 J. Stat. Phys. 36471

[14] Ramshaw J 1980 Mol. Phys. 41219

[15] White J A, Gonzalez A, Roman F L and Velasco S 2000 Phys. Rev. Lett. 84 1220; White J A and Velasco S, 2000 Phys. Rev. E 624427

[16] White J A and Velasco S 2000 Phys. Rev. E 624427

[17] Unruh K M, Huber T E and Huber C A 1993 Phys. Rev. B 48 9021; Duffy J A, Wilkinson N J, Fretwell H M, Alam M A and Evans R 1995 J. Phys. Condens. Matter 7 L713

[18] Evans R 1990 J. Phys. Condens. Matter 28989

[19] Neimark A, Rovitovitch P I and Vishnyakov A 2000 Phys. Rev. E 62 R1493

[20] Dominguez H, Allen M.A. and Evans R. 1999 Mol. Phys. 96209

[21] Rowlinson J S 1985 Proc. R. Soc. Lond. A 402 67; Rowlinson J S 1986 J. Chem. Soc. Faraday Trans. 282 1801; McQuarrie D A and Rowlinson J S 1987 Mol. Phys. 60977

[22] Woods G B, Panagiotopoulos A Z and Rowlinson J S 1988 Mol. Phys. 6349

[23] Gibbs J H, Bagchi B and Mohanty U 1981 Phys. Rev. B 242893

[24] Lee D J, Telo da Gama M M and Gubbins K E, J. Chem. Phys. 85490

[25] Kim S and Cummings P T 2001 Mol. Phys. 99 1099; Segura C J, Zhang J and Chapman W G 2001 Mol. Phys. 99 1; McGrother S.C. and Gubbins K E 1999 Mol. Phys. 97 955; Gelb L D and Gubbins K E 1999 Mol. Phys. 961795

[26] Gozdz W T, Gubbins K E and Panagiotopoulos A Z 1995 Mol. Phys. 84825

[27] Gubbins K E, Sliwinska-Barthkowiak M and Suh S 1997 Mol. Simul. 17333

[28] Hernando J A and Blum L 2001 J. Phys. Cond. Matter 13 L577

[29] Iyetomi H and Vashishta P 1989 J. Phys. Condens. Matt. 11899

[30] Hansen J P and McDonald I R 1990 Theory of Simple Liquids (New York: Academic Press) (2nd ed.)

[31] Lebowitz J L and Percus J K 1963 J. Math. Phys. 4116

[32] Lebowitz J L and Percus J K 1961 Phys. Rev. 1221675

[33] Wertheim M S 1967 J. Math. Phys. 8927

[34] Hernando J A 1986 Phys. Rev. A 331338

[35] Henderson J R 1987 Phys. Rev. A 364527 